\title{
Meddling with the mind
}

Individuals who suffer a stroke are at an increased risk of subsequently developing dementia, but the reasons for this are unclear. A new study in the Journal of Neuroscience by Marion Buckwalter and colleagues suggests that $B$ cells could be the missing link.

$B$ cells had not previously

B cells may contribute to the cognitive decline that is seen in some patients after stroke

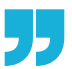

by a period of hypoxia. DH stroke produces a lesion adjacent to, but not incorporating, the hippocampus. Importantly, unlike other mouse models of stroke, DH stroke does not cause immediate cognitive impairment in mice.

When the authors assessed brain tissue from mice with DH stroke, they found that there is a delayed infiltration of B cells into stroke-induced lesions. Very few B cells could be detected in lesions 1 week after stroke, but there was marked infiltration of B cells into these sites at 7 weeks and 12 weeks. Histological analyses showed that B cells in the lesions colocalized with CD $11 \mathrm{c}^{+}$cells and T cells. Plasma cells were also present in the lesions and extensive antibody deposition was seen in both the stroke region and adjacent areas at 7 weeks after stroke. Additional experiments suggested that although antibody may have entered the brain following disruption of the blood-brain barrier, at least some of it was being produced locally by plasma cells in the lesion.

Most of the antibody found in the brain at 7 weeks after stroke induction was located in the hippocampus. Long-term potentiation (LTP; the process through which synaptic strength is persistently increased) is one of the main mechanisms involved in memory storage in the hippocampus. Notably, the antibody deposition that occurred after stroke induction was associated with impaired LTP that got progressively worse from 7 to 12 weeks. The impairment of LTP was also associated with short-term memory deficits in mice, as assessed by studies using Y mazes. Strikingly, $\mu$ MT mice, which lack mature B cells, did not experience LTP deficits or show cognitive impairment after stroke, despite developing lesions that were similar to those of wild-type mice in terms of size and $\mathrm{T}$ cell infiltrates. Furthermore, mice treated with B cell-depleting antibodies at 5 days after stroke induction were also protected from developing cognitive defects at 7 weeks.

Finally, the authors assessed B cell density and antibody levels in postmortem brain tissue from patients who had experienced stroke and dementia. B cells could be detected in the stroke core or adjacent regions of 19 of the 21 subjects assessed, and subjects with higher $\mathrm{B}$ cell densities in the brain showed stronger immunostaining for IgG. Of note, B cell densities were significantly increased in brain tissue from individuals in the stroke and dementia group compared with age-matched controls that had not been diagnosed with stroke or dementia.

This study suggests that B cells may contribute to the cognitive decline that is seen in some patients after stroke. As B cell-depleting antibodies are already approved for use in humans, it will be exciting to see whether these findings can be translated into the clinic.

Yvonne Bordon, Senior Editor, Nature Reviews Immunology This article is modified from the original in Nature Rev. Immunol. (http://dx.doi.org/10.1038/nri3826)

ORIGINAL RESEARCH PAPER Doyle, K. P. et al. B-lymphocyte-mediated delayed cognitive impairment following stroke. J. Neurosci. 35 , 2133-2145 (2015) 